\section{PSICOLOGÍA IBEROAMERICANA}

\section{Psicología Iberoamericana}

ISSN: 1405-0943

psicología.iberoamericana@uia.mx

Universidad Iberoamericana, Ciudad de México

México

Benítez Quintero, Fabiola; Cantón Cortés, David; Delgadillo Guzmán, Leonor Guadalupe Caracterización de la violencia sexual durante la infancia y la adolescencia

Psicología Iberoamericana, vol. 22, núm. 1, enero-junio, 2014, pp. 25-33

Universidad Iberoamericana, Ciudad de México

Distrito Federal, México

Disponible en: http://www.redalyc.org/articulo.oa?id=133944229004

- Cómo citar el artículo

- Número completo

- Más información del artículo

- Página de la revista en redalyc.org

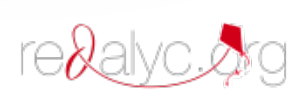

Sistema de Información Científica

Red de Revistas Científicas de América Latina, el Caribe, España y Portugal Proyecto académico sin fines de lucro, desarrollado bajo la iniciativa de acceso abierto 


\title{
Caracterización de la violencia sexual durante la infancia y la adolescencia
}

\author{
Characterization of sexual abuse during \\ infancy and adolescence
}

\author{
Fabiola Benítez Quintero ${ }^{1}$ \\ Universidad Autónoma del Estado de México \\ David Cantón Cortés ${ }^{2}$ \\ UnIVERSIDAD de MálaGA \\ Leonor Guadalupe Delgadillo Guzmán ${ }^{3}$ \\ Universidad Autónoma del Estado de México
}

\section{RESUMEN}

La presente investigación tuvo como objetivo establecer las características comunes de las agresiones sexuales vividas durante la niñez y la adolescencia en el municipio de Naucalpan, Estado de México. Se trabajó con una muestra compuesta por 38 niños y 40 adolescentes de ambos sexos, que reportaron haber sido víctimas de abuso sexual. Se realizó un procedimiento estadístico de tablas de contingencia para determinar los porcentajes de los indicadores de cada variable. Se encontró que la mayoría de las víctimas son mujeres que están en el inicio de la pubertad, la penetración vaginal forzada y los tocamientos son el primordial tipo de agresión, mientras que el principal agresor es aquel que representa a la figura paterna (padre o padrastro) y, por consiguiente, las agresiones suceden mayoritariamente en la casa donde conviven víctima y agresor. Los agresores regularmente son hombres con edades que oscilan entre los 21 y 30 años.

Palabras clave: violencia sexual, víctima, agresor, niños, adolescentes.

\section{ABSTRACT}

This research aimed to establish the common characteristics of sexual abuse experienced during childhood and adolescence in the municipality of Naucalpan in the State of Mexico. The study used a working sample of 38 boys and 40 adolescents who reported having been victims of sexual abuse. A statistical procedure was carried out using contingency tables to determine the percentages of the indicators of each variable. It was found that most of the victims are women reaching the age of puberty, and that forced penetration and unwelcome touching are the main types of abuse, while paternal figures (father or step-father) are the most common abusers and therefore the abuse generally takes place in the house shared by both: victim and abuser. Abusers are regularly men between 21 and 30 years old.

Keywords: sexual violence, victim, abuser, children, adolescents.

\footnotetext{
${ }^{1}$ Recibido: 20 de julio de 2013 - Aceptado: 6 de abril de 2014.

Licenciada Fabiola Benítez Quintero. Candidata a maestra en Psicología por la Universidad Autónoma del Estado de México.

Correo electrónico: fabiola_jego@hotmail.com

${ }^{2}$ Doctor David Cantón Cortés. Profesor de Psicología Evolutiva y de la Educación de la Universidad de Málaga.

Correo electrónico: david.canton@uma.es

${ }^{3}$ Doctora Leonor Guadalupe Delgadillo Guzmán. Profesora investigadora de la Universidad Autónoma del Estado de México.

Correo electrónico: delgadilloleonor@gmail.com
} 


\section{INTRODUCCIÓN}

La investigación en materia de abuso sexual infantil resulta una tarea difícil en el momento de revelar estadísticas objetivas, debido a que cultural y socialmente el tema se trata en forma secreta para los implicados en el acto. Del mismo modo, dentro de los tipos de maltrato infantil, se advierte que el abuso sexual en la infancia es el que ha suscitado mayor repercusión social, psicológica y política en los últimos años, dando lugar a una marcada dificultad para crear una delimitación del concepto, que no sólo se basa en la falta de acuerdo de los profesionales, sino también en las limitaciones legales en relación con la edad del menor (Mebarak, Martínez, Sánchez \& Lozano, 2010; Cantón \& Cortés, 2011).

Las consecuencias del abuso sexual en el caso de niños y adolescentes suelen relacionarse con el desarrollo de problemas de conducta, complicaciones familiares y problemas escolares, mientras que el impacto social se vincula con el aumento del presupuesto para el tratamiento integral de las víctimas y sus familias por parte de los sistemas de justicia (Cantón \& Cortés, 2008; Frías \& Gaxiola, 2008; Sanmartín, 2008).

Las estadísticas mundiales reflejan que dicho tema se ha convertido en un problema de salud pública. Diversos estudios internacionales revelan una prevalencia hasta de $20 \%$ para las mujeres y entre 5 y $10 \%$ para los varones (Ellsberg \& Heise, 2005). Por su lado, en lo que concierne al caso específico de México, el Instituto Mexicano del Seguro Social (IMss) reveló que durante el año 2010 se canalizaron a 18012 menores para realizar denuncias de maltrato infantil, de las cuales alrededor de $5 \%$ correspondieron a denuncias referentes a abuso sexual infantil, lo que significó cerca de novecientas denuncias al año, sólo en esta institución (Ramos, González, Toledo, Turrent \& Juárez, 2011).

Existen numerosas definiciones de abuso sexual infantil. Arredondo (2002) aporta una que contempla los elementos necesarios para su explicación sintética, refiriéndolo como:

Aquel tipo de conducta sexual que se realiza con un niño o niña, tales como tocamiento de los genitales u otras partes del cuerpo del niño por parte del agresor, incitación del perpetrador a tocar sus propios genitales, penetración vaginal, anal u oral, exposición de material pornográfico para el menor, utilización del infante para realización de material pornográfico. Estas situaciones pueden darse de manera conjunta o aislada, pueden ser efectuadas en una sola ocasión, varias veces o en abusos que se extiendan durante varios años (p. 6).

Del mismo modo, hay diferentes criterios que se han planteado para hacer una clasificación de los tipos de abuso sexual infantil. Una de ellos es el propuesto por Lago y Céspedes (2009):

- Con contacto físico: violación (penetración en la vagina, ano o boca con el pene o con cualquier objeto), caricias (tocar o acariciar los genitales del otro o cualquier otra parte del cuerpo con fines de satisfacción sexual).

- Sin contacto físico: propuestas verbales de actividad sexual explícita, mostrar los órganos sexuales de manera inapropiada, obligar a los niños a ver actividades sexuales de otras personas, falsas alegaciones de agresiones sexuales en contra de uno de los padres dentro de un proceso de divorcio.

- Explotación sexual: implicar a menores de edad en conductas o actividades relacionadas con la producción de pornografía, promover la prostitución infantil, el turismo sexual.

Por su parte, Ferreyra (2000) señala que la violencia sexual infantil puede darse por acción (refiriendo aquella implicación de un menor en situaciones sexuales para satisfacer las necesidades de personas adultas) y el maltrato sexual por omisión (haciendo énfasis en aquellas situaciones en que no se atienden las necesidades del niño en el área de la sexualidad, no se le da credibilidad en denuncias de situaciones de violencia sexual, se consiente de manera pasiva el incesto y no se educa sexualmente al menor).

Por otro lado, Acosta, Alva, Bueno, Díaz y Fernández (2007) establecen la presencia de tres condiciones necesarias dentro de la dinámica de abuso:

- Asimetría de poder: el ofensor controla a la víctima y la relación sexual no es concebida como algo mutuo. 
- Asimetría de conocimiento: el ofensor posee un conocimiento más sofisticado en el campo de la sexualidad.

- Asimetría de gratificación: el ofensor intenta obtener gratificación sexual exclusiva.

Las presentes condiciones han coadyuvado a comprender de mejor manera, que a comparación de otros tipos de violencia sexual, el abuso sexual en la infancia y en la adolescencia no se da sólo con base en la coerción y la fuerza, sino que también puede tener lugar por medio de la seducción. Esta situación sucede principalmente en familias donde los menores sufren de fuerte abandono emocional. Los agresores aprovechan esta situación para llenar las necesidades emocionales de abandono del menor, creando así el escenario que más adelante permitirá el abuso, y en el cual se facilitará que la víctima acceda al contacto sexual por miedo a perder el vínculo que ha generado con el perpetrador (Intebi, 2008).

A pesar de las complicaciones para crear estadísticas objetivas en torno al abuso sexual, varias investigaciones coinciden en que el vínculo con el agresor es muy variable en el tipo de relación: pueden ser extraños, pero también conocidos; $43 \%$ de las agresiones de abuso sexual infantil son cometidas por familiares y $33 \%$, por personas conocidas por la familia (López, 1999).

Por otro lado, y en lo que atañe a la violencia sexual en contra de los adolescentes, no existe una multiplicidad de estudios que proporcione diferentes perspectivas del problema; sin embargo, en algunos de los pocos que se han desarrollado, existen hallazgos relacionados con que las agresiones sexuales en este periodo vital ocurren frecuentemente en el marco de las relaciones de amistad o noviazgo, debido a que los jóvenes comienzan a aprender y ensayar nuevas formas de comportamiento acordes con su creciente libertad e independencia de la familia de origen. Por otra parte, son influenciados por la cultura inequitativa en la que se desarrollan, donde las mujeres son encasilladas como responsables de haber sido agredidas por no haber puesto límites pertinentes dentro del vínculo de pareja y acceder a tener relaciones sexuales por presiones o amenazas sin dar su entero consentimiento (Ortega, Ortega \& Sánchez, 2008; Rey, Mateus \& Bayona, 2010).
Aguilar y Salcedo (2008) mencionan que en el caso de los adolescentes, las mujeres que se encuentran entre los 10 y 14 años de edad son las que presentan mayor riesgo de vivir una agresión de esta índole y los principales agresores son personas pertenecientes o cercanas al entorno familiar. Asimismo, reportan que la amenaza, el chantaje y la inducción al silencio fueron las estrategias primordiales que usaron los perpetradores para abordarlas. Se destaca además la presencia de cómplices al momento del abuso.

Por lo tanto, y con base en lo explicado anteriormente, el objetivo del presente estudio fue establecer las características comunes y diferencias respecto a los datos sociodemográficos y condiciones que rodearon el evento de violencia sexual durante la infancia y la adolescencia en un grupo de víctimas, en el municipio de Naucalpan, Estado de México.

\section{MÉTODO}

\section{Muestra}

La muestra se integró con los datos obtenidos por medio de la revisión de los expedientes personales de 38 niños y niñas (con un promedio de 8.15 años de edad) y 40 adolescentes (con una media de 14.02 años de edad) que reportaron ser víctimas de abuso sexual e interpusieron una denuncia por dicho delito. Los diagnósticos se realizaron en la Unidad de Atención a Víctimas del Delito del municipio de Naucalpan, Estado de México, durante los años 2010 y 2011.

\section{Procedimiento}

Para realizar el análisis de ambos grupos se establecieron las siguientes variables: sexo, edad, escolaridad, ocupación, religión, nivel socioeconómico, estado civil de los padres, relación con el agresor, sexo del agresor, edad del agresor, lugar de la agresión, tipo de violencia sexual y continuidad del evento. Los embarazos no planeados, infecciones de trasmisión sexual o necesidad de hospitalización como resultado de la agresión fueron variables únicas de la muestra de adolescentes. Es importante mencionar que el expediente personal de cada una de las víctimas está integrado por un diagnóstico psicológico que recaba datos referentes al sexo, edad, escolaridad, ocupación, religión, breve historia de la composición familiar y antecedentes del caso, 
donde se relata de manera general la agresión referida por la persona. Asimismo, se integra un reporte que realiza el área de Trabajo Social, en el que se registran las condiciones socioeconómicas, así como la necesidad de canalizaciones médicas por embarazos no planeados, infecciones de transmisión sexual o alguna otra circunstancia que apremie hospitalización como resultado del ataque sexual.

Se procedió a revisar cada uno de los expedientes para obtener estos datos y computarlos en una base de datos en el programa spss Statistics 17.0, para que subsecuentemente fuera posible elaborar un análisis descriptivo por medio de tablas de contingencia, que arrojara las frecuencias y porcentajes de cada uno de los indicadores de las variables establecidas.

Considerando los principios básicos de la investigación ética, los datos se manejaron con apego a las premisas establecidas respecto a la confidencialidad de los resultados en los artículos 65 y 68 del Código Ético del Psicólogo (Sociedad Mexicana de Psicología, 2002) y contando con la autorización de la Procuraduría General de Justicia del Estado de México para tal efecto.

\section{RESULTADOS}

\section{Características sociodemográficas}

La mayor incidencia de víctimas de abuso sexual se encuentra en el rango de edad de 12 a 13 años para las adolescentes con un $16.7 \%$, y de 10 y 11 años para las niñas con un $15.4 \%$. El grupo de las mujeres presentó mayor frecuencia, constituyendo $84.6 \%$ del total de la muestra.

El nivel de escolaridad que presentan la mayoría de las víctimas en el grupo de niños se sitúa en aquellos que se encuentran cursando la primaria con $37.1 \%$, y en el grupo de los adolescentes, aquellos que cursan la secundaria con $16.7 \%$.

La mayor concentración de víctimas se localiza en el nivel socioeconómico medio para ambos grupos, constituyendo $26.9 \%$ para los niños y $34.6 \%$ para los adolescentes. El segundo porcentaje con mayor incidencia fue el nivel socioeconómico bajo con 20.5\% para los niños y $14.1 \%$ para los adolescentes.

En lo que respecta a las creencias religiosas, la mayor concentración se localizó en la religión católica para ambos grupos de edad, constituyendo $41.1 \%$ para el grupo de los niños y $43.5 \%$ para los adolescentes. El segundo porcentaje con mayor incidencia para los niños fue la congregación de los testigos de Jehová, con un $5.2 \%$, mientras que para los adolescentes fue el ateísmo, con un 3.9\%.

La categoría estudiante obtuvo mayores índices para ambos grupos de edad, constituyendo $46.1 \%$ para los niños y $29.5 \%$ para los adolescentes. La segunda con mayor concentración fue ninguna ocupación con $2.6 \%$ para el grupo de los niños y, finalmente, la ocupación de ama de casa para las adolescentes, con $12.9 \%$

Tabla 1. Contingencia por edad y sexo de las víctimas

\begin{tabular}{|c|c|c|c|c|c|c|}
\hline \multirow{2}{*}{ Edad } & \multicolumn{2}{|c|}{ Mujeres } & \multicolumn{2}{|c|}{ Hombres } & \multicolumn{2}{|c|}{ Total } \\
\hline & Casos & Porcentaje & Casos & Porcentaje & Casos & Porcentaje \\
\hline 4-5 años & 5 & $6.4 \%$ & 3 & $3.8 \%$ & 8 & $10.3 \%$ \\
\hline 6-7 años & 8 & $10.3 \%$ & 3 & $3.8 \%$ & 11 & $14.1 \%$ \\
\hline 8-9 años & 7 & $9 \%$ & 2 & $2.6 \%$ & 9 & $11.5 \%$ \\
\hline 10-11 años & 10 & $12.8 \%$ & 2 & $2.6 \%$ & 12 & $15.4 \%$ \\
\hline 12-13 años & 12 & $15.4 \%$ & 1 & $1.3 \%$ & 13 & $16.7 \%$ \\
\hline 14-15 años & 10 & $12.8 \%$ & 0 & $0 \%$ & 10 & $12.8 \%$ \\
\hline 16-17 años & 11 & $14.1 \%$ & 1 & $1.3 \%$ & 12 & $15.4 \%$ \\
\hline 18-19 años & 3 & $3.8 \%$ & 0 & $0 \%$ & 3 & $3.8 \%$ \\
\hline Total & 66 & $84.6 \%$ & 12 & $15.4 \%$ & 78 & $100 \%$ \\
\hline
\end{tabular}


La mayor concentración en el grupo de niños se ubicó en la categoría de las madres que viven con una pareja diferente al padre (familia reconstruida) con $17.9 \%$, seguida de los padres separados y por los padres casados con $15.3 \%$ cada una de estas categorías.

En el caso de los adolescentes, la mayor concentración se localizó en los padres casados 16.6\%, seguido de los padres separados con $12.8 \%$.

\section{Características del abuso, víctima y agresor}

En el grupo de los niños, la persona que resultó ser el agresor con mayor frecuencia fue el padrastro con $9 \%$, seguido por el tío con $7.7 \%$ y los vecinos igualmente con $7.7 \%$. En el caso de los adolescentes, se reportó como principal perpetrador a la figura paterna con $12.8 \%$ (6.4\% para el padre y $6.4 \%$ para el padrastro), seguidos de las personas desconocidas con $7.7 \%$.
En lo que respecta al sexo y edad del agresor, la mayor concentración se encontró en primer lugar en hombres de 21 a 30 años de edad con un porcentaje de $21.4 \%$ para el grupo de niños y $14.1 \%$ para el grupo de adolescentes. En segundo lugar de concentración de porcentaje se encuentran los hombres de 31 a 40 años para ambos grupos, constituyendo $15.45 \%$ para el caso de los niños y en el caso de los adolescentes, $10.2 \%$.

En cuanto al lugar en el que sucedió la agresión, la mayor concentración del porcentaje se registró en primer lugar en la casa de ambos para los dos grupos, con un porcentaje de $19.3 \%$, para los niños y con $10.2 \%$ para el grupo de adolescentes. En segundo lugar de concentración de porcentaje se encontró la casa del agresor con $16.7 \%$ para los niños y niñas, mientras que para el grupo de adolescentes se reportó la casa de la víctima con $9 \%$.

Tabla 2. Tabla de contingencia por grupo de edad y relación con el agresor

\begin{tabular}{|c|c|c|c|c|c|c|}
\hline \multirow{2}{*}{ Relación con el agresor } & \multicolumn{2}{|c|}{ Niños } & \multicolumn{2}{|c|}{ Adolescentes } & \multicolumn{2}{|c|}{ Total } \\
\hline & Casos & Porcentaje & Casos & Porcentaje & Casos & Porcentaje \\
\hline Padre & 3 & $3.8 \%$ & 5 & $6.4 \%$ & 8 & $10.3 \%$ \\
\hline Padrastro & 7 & $9 \%$ & 5 & $6.4 \%$ & 12 & $15.4 \%$ \\
\hline Madrastra & 2 & $2.6 \%$ & 0 & $0 \%$ & 2 & $2.6 \%$ \\
\hline Tío & 6 & $7.7 \%$ & 2 & $2.6 \%$ & 8 & $10.3 \%$ \\
\hline Hermano & 2 & $2.6 \%$ & 1 & $1.3 \%$ & 3 & $3.8 \%$ \\
\hline Abuelastro & 3 & $3.8 \%$ & 0 & $0 \%$ & 10 & $3.8 \%$ \\
\hline Primo & 3 & $3.8 \%$ & 1 & $1.3 \%$ & 4 & $5.1 \%$ \\
\hline Cuñado & 0 & $0 \%$ & 0 & $0 \%$ & 1 & $1.3 \%$ \\
\hline Amigo & 0 & $0 \%$ & 3 & $3.8 \%$ & 3 & $3.8 \%$ \\
\hline Novio & 0 & $0 \%$ & 3 & $3.8 \%$ & 3 & $3.8 \%$ \\
\hline Ex novio & 0 & $0 \%$ & 1 & $1.3 \%$ & 1 & $1.3 \%$ \\
\hline Conocido de la familia & 3 & $3.8 \%$ & 2 & $2.6 \%$ & 5 & $6.4 \%$ \\
\hline Vecino & 6 & $7.7 \%$ & 3 & $3.8 \%$ & 9 & 11.5 \\
\hline Profesor & 2 & $2.6 \%$ & 2 & $2.6 \%$ & 4 & $5.1 \%$ \\
\hline Desconocido & 0 & $0 \%$ & 7 & $7.7 \%$ & 6 & $7.7 \%$ \\
\hline Otro & 3 & $3.8 \%$ & 2 & $2.6 \%$ & 5 & $6.4 \%$ \\
\hline Total & 40 & $51.3 \%$ & 38 & $48.7 \%$ & 78 & $100 \%$ \\
\hline
\end{tabular}


Tabla 3. Tabla de contingencia por grupo de edad y lugar de la agresión

\begin{tabular}{|c|c|c|c|c|c|c|}
\hline \multirow{2}{*}{ Lugar de la agresión } & \multicolumn{2}{|c|}{ Niños } & \multicolumn{2}{|c|}{ Adolescentes } & \multicolumn{2}{|c|}{ Total } \\
\hline & Casos & Porcentaje & Casos & Porcentaje & Casos & Porcentaje \\
\hline Casa de la víctima & 4 & $5.1 \%$ & 7 & $9 \%$ & 11 & $14.1 \%$ \\
\hline Casa del agresor & 13 & $16.7 \%$ & 4 & $5.1 \%$ & 17 & $21.8 \%$ \\
\hline Casa de ambos & 15 & $19.3 \%$ & 8 & $10.2 \%$ & 23 & $29.5 \%$ \\
\hline Casa de desconocidos & 0 & $0 \%$ & 6 & $7.7 \%$ & 6 & $7.7 \%$ \\
\hline Escuela & 3 & $3.8 \%$ & 1 & $1.3 \%$ & 4 & $5.1 \%$ \\
\hline Automóvil & 1 & $1.3 \%$ & 2 & $2.6 \%$ & 3 & $3.8 \%$ \\
\hline Transporte público & 0 & $0 \%$ & 2 & $2.6 \%$ & 2 & $2.6 \%$ \\
\hline Calle & 1 & $1.3 \%$ & 2 & $2.6 \%$ & 3 & $3.8 \%$ \\
\hline Bosque & 0 & $0 \%$ & 2 & $2.6 \%$ & 2 & $2.6 \%$ \\
\hline Otro & 3 & $3.8 \%$ & 4 & $5.1 \%$ & 7 & $9 \%$ \\
\hline Total & 40 & $51.3 \%$ & 38 & $48.7 \%$ & 78 & $100 \%$ \\
\hline
\end{tabular}

Tabla 4. Tipo de agresión sexual*

\begin{tabular}{l|c|c|c|c|c|c}
\hline \multirow{2}{*}{ Tipo de agresión } & \multicolumn{2}{|c|}{ Niños } & \multicolumn{2}{c}{ Adolescentes } & \multicolumn{2}{c}{ Total } \\
\cline { 2 - 7 } & Casos & Porcentaje & Casos & Porcentaje & Casos & Porcentaje \\
\hline Voyeurismo/exhibicionismo & 1 & $1.3 \%$ & 2 & $2.6 \%$ & 3 & $3.9 \%$ \\
\hline Tocamientos & 20 & $25.6 \%$ & 14 & $18 \%$ & 34 & $43.6 \%$ \\
\hline Sexo oral & 12 & $15.4 \%$ & 2 & $2.6 \%$ & 14 & $18 \%$ \\
\hline Penetración vaginal & 14 & $18 \%$ & 29 & $37.1 \%$ & 43 & $55.1 \%$ \\
\hline Penetración anal & 16 & $20.5 \%$ & 3 & $3.9 \%$ & 19 & $24.4 \%$ \\
\hline Violación tumultuaria & 1 & $1.3 \%$ & 2 & $2.6 \%$ & 3 & $3.9 \%$ \\
\hline
\end{tabular}

* La tabla 4 no presenta la suma de los porcentajes debido a que, en algunos casos, cada uno de los participantes fue víctima de más de un tipo de agresión.

El tipo de agresión sexual que se reportó con mayor frecuencia en el grupo de niños fueron los tocamientos (25.6\%), seguido de la penetración vaginal forzada (18\%). En lo que respecta al grupo de adolescentes, la agresión que se registró con más incidencia fue la penetración vaginal forzada (37.1\%), seguida por los tocamientos (18\%).
En el grupo de los niños, $23.2 \%$ reportaron haber sido víctima de abuso en una sola ocasión, seguido de la categoría de haber sufrido abusos continuados, con 20.4\%. En el caso de los adolescentes, se concentró con $28.1 \%$ una agresión aislada, seguida de la categoría de abusos continuados con $12.8 \%$. 
Tabla 5. Continuidad del abuso

\begin{tabular}{l|c|c|c|c|c|c}
\hline \multirow{2}{*}{ Continuidad } & \multicolumn{2}{|c|}{ Niños } & \multicolumn{2}{c|}{ Adolescentes } & \multicolumn{2}{c}{ Total } \\
\cline { 2 - 7 } & Casos & Porcentaje & Casos & Porcentaje & Casos & Porcentaje \\
\hline Una ocasión & 18 & $23.2 \%$ & 22 & $28.1 \%$ & 40 & $51.3 \%$ \\
\hline Varias ocasiones & 6 & $7.8 \%$ & 6 & $7.8 \%$ & 12 & $15.4 \%$ \\
\hline Agresión continuada & 16 & $20.4 \%$ & 10 & $12.8 \%$ & 26 & $33.2 \%$ \\
\hline Total & 40 & $51.3 \%$ & 38 & $48.7 \%$ & 78 & $100 \%$ \\
\hline
\end{tabular}

La consecuencia más común de la violencia sexual hacia las adolescentes es la maternidad no planeada, con $10.52 \%$ de incidencia, seguida de las infecciones de transmisión sexual y desgarres genitales, con 3.8\% cada uno.

\section{DISCUSIÓN}

El presente estudio permitió caracterizar la violencia sexual en un grupo de niños y en uno de adolescentes en el Estado de México, se encontró por un lado el reforzamiento de datos que se han publicado desde hace varios años, relacionados con que la gran mayoría de víctimas son mujeres (tanto niñas como adolescentes) y el más alto porcentaje de perpetradores son hombres de entre 21 y 30 años (Acosta et al., 2007; Mebarak et al., 2010; Perrone \& Nannini, 2005). Por otro lado, se ubicaron indicadores novedosos vinculados con que las mujeres adolescentes son más propensas a sufrir penetración vaginal forzada como principal tipo de agresión, mientras que en el grupo de los niños son los tocamientos. Asimismo, se encontró que el lugar donde suceden con más frecuencia los abusos sexuales es la casa donde conviven la víctima y el agresor.

El grupo más afectado por esta problemática de salud pública son las mujeres adolescentes que se encuentran al inicio de esta etapa, como resultado de que muestran claros indicadores de desarrollo sexual, dándole apariencia física de mujer; pero en lo que respecta a su desarrollo psicológico y social aún no han adquirido habilidades sociales y de afrontamiento que les ayuden a detectar que están siendo víctimas de abuso, y si lo identifican, optan por guardar silencio porque no saben cómo ni con quién deben manejar la situación, lo que genera sentimientos de culpa hacia sí mismas (Finkelhor, 2005; Intebi, 2008).

La mayor concentración de víctimas se ubicó en el nivel socioeconómico medio, seguida por el nivel socioeconómico bajo. Perrone y Nannini (2005) señalan que los abusos sexuales suceden en todos los niveles; sin embargo, las estadísticas en el nivel socioeconómico alto no llegan a ser significativas, debido a que buscan apoyo legal y psicológico de manera privada, ya que algunos de ellos son figuras públicas (artistas, políticos, empresarios).

La escuela es un lugar importante para la búsqueda de apoyo y soporte por parte de las víctimas de abuso sexual, debido a que la mayoría de ellas en esta muestra se encuentran cursando el nivel básico de educación; por lo tanto, se considera necesario orientar el tema de prevención de abuso sexual dentro de los contextos escolares, para los menores, los docentes y los padres de familia, a fin de crear una red de apoyo para que sea posible prevenir a los más vulnerables (Aguilar \& Salcedo, 2008).

En lo relacionado con la religión, se observó una fuerte tendencia hacia el catolicismo, probablemente como resultado de que México es un país fundamentalmente católico, basado en supuestos valores universales y trascendentales (Pérez-Rayón, 2006), lo que puede estar relacionado con las creencias que plantean algunos dogmas de fe acerca de la prohibición de los temas vinculados con la sexualidad, el tabú y distorsión de la información que en ocasiones se transmite en este ámbito (De la Rubia, 2010).

Respecto a la composición familiar, se encontró que el grupo de niños que han sido agredidos provie- 
nen en su mayoría de familias reconstruidas, donde la madre tiene una nueva pareja. Dicha situación corresponde con el dato arrojado en la presente investigación que señala al padrastro como principal agresor de este grupo de edad. Lo anterior coincide con Horno, Del Molino y Santos (2001), quienes mencionan que en América Latina 55\% de las violaciones a niños y niñas son intrafamiliares, perpetradas por el padre, el padrastro y otros parientes. El segundo porcentaje de incidencia de agresores lo tuvieron los tíos y los vecinos y el lugar en que se reportaron mayoritariamente las agresiones fueron la casa donde conviven la víctima y el agresor y la casa del agresor. Pereda, Polo, Grau, Navales y Martínez (2007) informan que los principales perpetradores son personas conocidas y, por lo tanto, cercanos, el principal puntaje lo tienen aquellas que desempeñan el papel de figura paterna, ya sea el padre biológico, el padrastro o algún tío o abuelo que cumpla con dicha función.

En cuanto a la estructura familiar de los adolescentes, se encontró que la mayoría de ellos provienen de familias integradas en las que ambos padres están juntos. Asimismo, se observó que el principal agresor de este grupo es la persona que ejerce el papel de figura paterna y la casa donde viven ambos fue el lugar en el que se suscitaron más frecuentemente las agresiones.

Las personas desconocidas ocuparon el segundo lugar en frecuencia de figuras perpetradoras para los adolescentes. Fue complicado corroborar esta situación, ya que existen muy pocos estudios centrados en la violencia sexual durante la adolescencia en México, debido a que en las investigaciones realizadas, tiende a situárseles en el grupo de niños o de adultos, pero en raras ocasiones se hace referencia a ellos como conjunto específico (Sullivan, 1997; Cantón \& Cortés, 2008).

El mayor porcentaje de los agresores continúa siendo de hombres, con una mayor concentración en el rango de edad de 21 a 30 años, lo cual coincide con la etapa de la vida de los hombres en la que tienen mayor actividad sexual y desarrollo de fuerza física (Ysern de Arce \& Becerra, 2006).

Por último, el tipo de violencia sexual que resultó más frecuente en el grupo de los niños fueron los tocamientos, seguido de la penetración anal forzada. En el caso de los adolescentes, el tipo de agresión que presentó mayor incidencia fue la penetración vaginal forzada, seguida de los tocamientos. Probablemente esta situación se encuentre relacionada con que las adolescentes muestran signos más claros de desarrollo sexual y como resultado de esta situación, muchas de las víctimas tienen que enfrentarse a maternidades no planeadas.

\section{CONCLUSIONES}

El abuso sexual infantil constituye un verdadero problema de salud pública, y el grupo que resulta más afectado es el de las mujeres, especialmente las que se encuentran en el inicio de la pubertad, entre los 10 y 13 años de edad (Ferreyra, 2000).

Las figuras agresoras se concentran principalmente en hombres de 21 a 30 años que forman parte del círculo familiar y social más cercano de las agredidas (López, 1999), sobre todo aquellos que representan a la figura paterna, dando lugar a un ambiente que facilita el abuso repetitivo, ya que se comete al interior de la casa que comparten el agresor y la víctima, lo cual genera indefensión en esta última, y permite que el abuso permanezca oculto a la vista de los demás.

En el caso de los niños y las niñas, los perpetradores identifican en sus víctimas la falta de habilidades sociales por la etapa de desarrollo en que se encuentran los menores; dicha carencia en las víctimas les dificulta identificar y revelar el abuso, por lo que esta dinámica puede extenderse durante largos periodos dentro del mismo espacio que comparten el agresor y la víctima (Cantón \& Cortés, 2008).

En lo que se refiere a los adolescentes, se debe subrayar la necesidad de crear estudios respecto a la violencia sexual focalizados en este grupo de edad, ya que si bien comparten ciertas condiciones y consecuencias con el conjunto de niños, también es importante distinguir que se encuentran en un periodo vital diferente y sus consecuencias físicas, psicológicas y sociales cambian (Ysern de Arce \& Becerra, 2010), debido a que en las adolescentes se suma la probabilidad de maternidades no planeadas ni deseadas. Por otro lado, a diferencia de los agresores de los niños, que en su mayoría se encuentran entre los círculos familiares y sociales más cercanos, en el caso de los adolescentes se reveló un alto índice de agresiones cometidas por personas desconocidas. 
Es necesario reformular las políticas públicas en lo que se vincula con el tema de educación sexual dentro de los centros escolares, debido a que la vulnerabilidad a convertirse en víctima de abuso sexual secreto y

\section{REFERENCIAS}

Acosta, M., Alva, A., Bueno, C., Díaz, N. \& Fernández, U. (2007). Prevención de la violencia sexual. México: Colección Mayor, Gobierno del Estado de México.

Aguilar, A. \& Salcedo, M. (2008). Caracterización de la violencia sexual en adolescentes de 10 a 19 años. Colombia Médica, 39(4), 356-363.

Arredondo, V. (2002). Guía básica de prevención del abuso sexual infantil. Paicabí, Chile: Gobierno de Chile.

Cantón, J. \& Cortés, M. (2008). Guía para la evaluación del abuso sexual infantil. Madrid: Pirámide.

Cantón, J. \& Cortés, M. (2011). Malos tratos y abuso sexual infantil. Causas, consecuencias e intervención. Madrid: Siglo XXI.

De la Rubia, J. (2010). Religión, significados y actitudes hacia la sexualidad: un enfoque psicosocial. Revista Colombiana de Psicología, 19, 45-49.

Ellsberg, M. \& Heise, L. (2005). Researching Violence Against women. Ginebra, Suiza: Organización Mundial de la Salud.

Ferreyra, S. (2000). Seminario de prevención de abuso sexual infantil en el ámbito educativo. Dinamarca: Asociación de Padres Danesa.

Finkelhor, D. (2005). Abuso sexual al menor. México: Pax. Frías, M. \& Gaxiola, J. (2008). Consecuencias de la violencia familiar experimentada directa e indirectamente en niños: depresión, ansiedad, conducta antisocial y ejecución académica. Revista Mexicana de Psicología, 25, 237-248.

Horno, P., Del Molino, A. \& Santos. A. (2001). Abuso sexual infantil: formación para profesionales. España: Save the children.

Intebi, I. (2008). Abuso sexual infantil en las mejores familias. Buenos Aires, Argentina: Garnica.

Lago, C. \& Céspedes, J. (2009). Abuso sexual infantil. Precop, 5, 16-30.

López, F. (1999). La inocencia rota: abuso sexual a menores. España: Océano. repetitivo aumenta durante la niñez y la adolescencia; por lo tanto, es de suma importancia trabajar de manera especializada la prevención de la violencia sexual en estas etapas de la vida.

Mebarak, M., Martínez, M., Sánchez, A. \& Lozano, J. (2010). Una revisión de la sintomatología del abuso sexual infantil. Psicología desde el Caribe, 25, 128154.

Ortega, R., Ortega, F. \& Sánchez, V. (2008). Violencia sexual entre compañeros y violencia en parejas adolescentes. International Journal of Psychology and Psychological Therapy, 8, 63-72.

Pereda, P., Polo, P., Grau, N., Navales, N. \& Martínez, M. (2007). Víctima de abuso sexual en la infancia: un estudio descriptivo. Revista de Estudios de la Violencia, $1,1-4$.

Pérez- Rayón, N. (2006). Iglesia católica, estado y narcotráfico. Un desafío hacia el siglo xxI. Revista Sociológica, 62, 139-173.

Perrone, R. \& Nannini, M. (2005). Violencia y abusos sexuales en la familia Buenos Aires, Argentina: Paidós.

Ramos, R., González, R., Toledo, G., Turrent, G. \& Juárez, M. (2011). Detección temprana del abuso físico desde el nacimiento hasta los 12 años de edad. México: Centro Nacional de Excelencia Tecnológica en Salud.

Rey, C., Mateus, A. \& Bayona, A. (2010). Malos tratos ejercidos por adolescentes durante el noviazgo: diferencias por sexo. Revista Mexicana de Psicología, 27, 169-181.

Sanmartín, J. (2008). Violencia contra niños. Barcelona, España: Ariel.

Sociedad Mexicana de Psicología (2002). Código ético del psicólogo. México: Trillas.

Sullivan, D. (1997). El sexo que se calla. México: Pax.

Ysern de Arce, J. \& Becerra, P. (2006). Abuso sexual intrafamiliar: prevalencia y características en jóvenes de tercer grado de liceos municipalizados de Chillan, Chile. Theoria, 15, 79-85. 